\title{
The use of milk mid-infrared spectroscopy to improve genomic prediction accuracy of serum biomarkers
}

\author{
I. van den Berg, ${ }^{1 *} \odot$ P. N. Ho, ${ }^{1} \odot$ T. D. W. Luke, ${ }^{1,2} \odot$ M. Haile-Mariam, ${ }^{1}$ S. Bolormaa, ${ }^{1}$ and J. E. Pryce ${ }^{1,2} \odot$ \\ ${ }^{1}$ Agriculture Victoria Research, AgriBio, Centre for AgriBioscience, 5 Ring Road, Bundoora, Victoria 3083, Australia \\ ${ }^{2}$ School of Applied Systems Biology, La Trobe University, Bundoora, Victoria 3083, Australia
}

\begin{abstract}
Breeding objectives in the dairy industry have shifted from being solely focused on production to including fertility, animal health, and environmental impact. Increased serum concentrations of candidate biomarkers of health and fertility, such as $\beta$-hydroxybutyric acid (BHB), fatty acids, and urea are difficult and costly to measure, and thus limit the number of records. Accurate genomic prediction requires a large reference population. The inclusion of milk mid-infrared (MIR) spectroscopic predictions of biomarkers may increase genomic prediction accuracy of these traits. Our objectives were to (1) estimate the heritability of, and genetic correlations between, selected serum biomarkers and their respective MIR predictions, and (2) evaluate genomic prediction accuracies of either only measured serum traits, or serum traits plus MIR-predicted traits. The MIR-predicted traits were either fitted in a single trait model, assuming the measured trait and predicted trait were the same trait, or in a multitrait model, where measured and predicted trait were assumed to be correlated traits. We performed all analyses using relationship matrices constructed from pedigree (A matrix), genotypes ( $\mathbf{G}$ matrix), or both pedigree and genotypes (H matrix). Our data set comprised up to 2,198 and 9,657 Holstein cows with records for serum biomarkers and MIR-predicted traits, respectively. Heritabilities of measured serum traits ranged from 0.04 to 0.07 for BHB, from 0.13 to 0.21 for fatty acids, and from 0.10 to 0.12 for urea. Heritabilities for MIR-predicted traits were not significantly different from those for the measured traits. Genetic correlations between measured traits and MIR-predicted traits were close to 1 for urea. For BHB and fatty acids, genetic correlations were lower and had large standard errors. The inclusion of MIR predicted urea substantially increased prediction accuracy for urea. For BHB, including MIR-predicted
\end{abstract}

Received August 12, 2020.

Accepted October 7, 2020.

*Corresponding author: irene.vandenberg@agriculture.vic.gov.au
BHB reduced the genomic prediction accuracy, whereas for fatty acids, prediction accuracies were similar with either measured fatty acids, MIR-predicted fatty acids, or both. The high genetic correlation between urea and MIR-predicted urea, in combination with the increased prediction accuracy, demonstrated the potential of using MIR-predicted urea for genomic prediction of urea. For BHB and fatty acids, further studies with larger data sets are required to obtain more accurate estimates of genetic correlations.

Key words: biomarkers, mid-infrared spectroscopy, genomic prediction, heritability, genetic correlation

\section{INTRODUCTION}

Breeding objectives in the dairy industry have shifted from being solely focused on production to focusing on improving fertility, animal health, and environmental impact (Boichard and Brochard, 2012). Increased serum concentrations of metabolites such as $\beta$-hydroxybutyric acid (BHB), fatty acids, and urea are promising biomarkers that are associated with reduced health, production, and fertility (Butler et al., 1996; Ospina et al., 2010a,b; Pryce et al., 2016; Raboisson et al., 2017). However, serum concentrations of these biomarkers are difficult and costly to measure and stressful to the animals, especially in the case of genomic selection, where a large reference population with recoded phenotypes is needed for accurate prediction. Recently, Luke et al. (2019a) published accuracies of $0.29,0.36$, and 0.30 for genomic prediction of fatty acids, BHB, and urea, respectively, using a data set of 1,393 Australian Holstein cows. As collecting additional phenotypes is logistically challenging, opportunities to dramatically increase the number of phenotypes in the near future is not realistic, and alternatives should be explored.

Mid-infrared (MIR) spectroscopy has routinely been used to estimate milk composition in the dairy industry. Mid-infrared spectral data is potentially available on all cows with production records, meaning that it could be an excellent way to generate numerous phenotypes. Many authors have reported promising accuracies when 
using milk MIR to predict these serum biomarkers, with the value of $\mathrm{R}^{2}$ obtained through random crossvalidation ranging between 0.21 and 0.92 (Belay et al., 2017; Grelet et al., 2019; Luke et al., 2019a,b; Pralle and White, 2020). In Australia, the equations for predicting serum BHB, fatty acids, and urea were initially developed by Luke et al. (2019b) and have recently been validated by Ho et al. (2020), with accuracies obtained through 10-fold random cross-validation of $0.60,0.42$, and 0.87 , respectively. In this context, if MIR-predicted biomarkers are heritable, genetically correlated with the measured serum biomarkers concentrations, and available on a large number of individuals, they should theoretically help to increase the size of reference populations, and thereby aid the implementation of genomic prediction for health traits based on biomarkers. Traits predicted by MIR can be fitted in a single trait model (assuming the measured trait and the predicted trait are the same trait), or in a multitrait model where the measured and MIR-predicted traits are assumed to be different, but correlated traits.

Genomic prediction requires animals in the reference population to have both phenotypes and genotypes; however, while the number of genotyped animals has increased, not all phenotyped cows are genotyped. Because of the small number of records for measured serum biomarkers, it might be preferable to use all phenotyped cows in prediction analyses, including cows that are not genotyped but have pedigree information recorded. This is possible with single step (ss) genomic BLUP (GBLUP; Aguilar et al., 2010). Furthermore, inclusion of nongenotyped individuals may reduce potential bias caused by preselection of genotyped individuals (Patry and Ducrocq, 2011; Misztal et al., 2020). While ssGBLUP is appealing because of the inclusion of ungenotyped individuals, GBLUP is currently used for routine genomic evaluation in Australia. Therefore, we compared estimates of genetic parameters and prediction accuracies using BLUP, GBLUP, and ssGBLUP.

The objectives of our study were to (1) estimate heritability of, and genetic correlations between, measured serum biomarkers and their MIR predictions, (2) estimate the accuracy of genomic prediction for biomarkers using either only measured serum traits, only MIR-predicted traits, or measured and MIR-predicted traits, and (3) compare genetic parameters and prediction accuracies using BLUP, GBLUP, and ssGBLUP.

\section{MATERIALS AND METHODS}

\section{Phenotypes}

Data set 1 included 3,027 Holstein-Friesian cows from 19 dairy herds in southeastern Australia that had both actual biomarkers measured in serum and predicted from MIR spectra. Between July 2017 and April 2020, blood samples were taken according to the protocol described in Luke et al. (2019b). The cows were between first and eighth parity. At the time of sampling, all cows were between 0 and 50 DIM, with a mean of $21.4 \pm 18.7$. All procedures were conducted in accordance with the Australian Code of Practice for the Care and Use of Animals for Scientific Purposes (NHMRC, 2013). Approval to proceed was granted by the Agricultural Research and Extension Animal Ethics Committee of the Department of Jobs, Precincts and Resources Animal Ethics Committee (Attwood, Victoria, Australia), and the Tasmanian Department of Primary Industries, Parks, Water and Environment (Animal Biosecurity and Welfare Branch, New Town, Tasmania, Australia). The sera were analyzed for concentrations of BHB, fatty acids, and urea by Regional Laboratory Services (Benalla, Victoria, Australia). On the same day of blood sampling, milk samples were collected and sent to Hico Pty Ltd. (Maffra, Victoria, Australia) or TasHerd Pty Ltd. (Hadspen, Tasmania, Australia) for analysis of fat, protein, and lactose percentages, as well as SCC using a NexGen Series FTS Combi machine (Bentley Instruments, Chaska, MN), from where the MIR spectra were obtained for this study.

The second data set comprised 7,932 animals from 51 herds that were different from those in data set 1 . These animals only had MIR data collected through the routine herd-testing program operated by Hico Pty Ltd. The cows calved between July 2016 and November 2019 with an average parity of 2.9. Days in milk varied between 0 and 50 .

The MIR-predicted biomarkers were derived using the equations previously developed by our group (Luke et al., 2019b; Ho et al., 2020). A full description of the development of the MIR equations used in our study can be found in Ho et al. (2020). Generally, the prediction accuracies $\left(\mathrm{R}^{2}\right)$ obtained for BHB, fatty acids, and urea were $0.60,0.42$ and 0.87 through 10 -fold random cross-validation, and $0.48,0.35$, and 0.69 through herdby-herd validation. Several mathematical treatments were applied to the biomarkers and the spectra data before performing the model predictions. For BHB and fatty acids, a logarithmic (10) and a square root transformation were applied to their original records, respectively. The spectral treatments included removal of regions $\left(1,615-1,552 \mathrm{~cm}^{-1}\right.$ and $\left.659-925 \mathrm{~cm}^{-1}\right)$ characterized by a low signal to noise ratio as a consequence of a high water absorption, removal of a noninformative region $\left(2,998-3,998 \mathrm{~cm}^{-1}\right)$, elimination of outliers based on Mahalanobis distance, and application of a Savitzky-Golay first derivative (De Marchi et al., 2014). 
For data set 1, MIR predictions were derived using an external herd-by-herd procedure, whereby the data of a given herd for which the predictions were to be obtained was first excluded, and subsequently predicted, using a model trained with the data from the remaining herds. The process was repeated until every herd had been predicted (i.e., 19 times, as there were 19 herds in data set 1). The MIR-predicted biomarkers in data set 2 were obtained by applying the prediction equations developed using records of data set 1 . All the predictions were performed using partial least squares regression, implemented with the pls $\mathrm{R}$ package of Mevik and Wehrens (2007).

For the genetic analyses, we combined data set 1 and data set 2, and removed 11 animals born before 2004 and 2,616 animals with unknown parity from the combined data set. This resulted in a total of 10,081 animals used in any of our analyses. Three different relationship matrices were used in our analyses. All animals with pedigree $(\mathbf{A})$, genotype $(\mathbf{G})$, or either pedigree or genotype $(\mathbf{H})$ were used in the analyses using the A matrix, $\mathbf{G}$ matrix, and $\mathbf{H}$ matrix, respectively. The number of records used in each analysis and the mean and standard deviation for each trait are summarized in Table 1.

\section{Genotypes}

Out of 7,053 cows with genotypes and measured biomarkers or MIR-predicted biomarkers, 123 cows were genotyped on the Illumina Bovine 50K panel (Illumina Inc., San Diego, CA) and the remaining were genotyped on a variety of low density (LD) chips ranging in size from approximately 8,500 SNP to 25,000 SNP. There was generally an overlap of at least 7,000 SNP between the LD panels and the 50K panel. All SNP positions were mapped to the ARS-UCD1.2 reference genome, and all variants with unknown chromosomes or positions were removed. Raw genotypes were filtered using the Illumina GenCall score: SNP were excluded if the call rate per SNP (this is the proportion of SNP genotypes that have a GenCall score above 0.6) was less than $95 \%$. Also, animals with less than $90 \%$ of their SNP genotypes with GenCall score $>0.6$ and their genotypes with excessive heterozygosity $(>0.4)$ were removed. The genotypes with GenCall score $<0.6$ were set as missing and then the sporadic missing values were filled using Fimpute v3 (Sargolzaei et al., 2014).

The genotypes for each SNP of both LD and 50k SNP panels were encoded in the Illumina TOP/TOP format and then genotypes were reduced to 0,1 , and 2 copies of the B allele. Imputation from LD SNP panels to the Illumina Bovine HD BeadChip panel (HD) was carried out using Fimpute v3 (Sargolzaei et al., 2014) in 2 sequential stages. Animals with LD genotypes were first imputed to the 50K SNP panel using a reference population of 14,722 animals and then to the HD panel using a reference population of 2,700 animals that were genotyped directly with the HD panel. After quality control described above, 575,704 SNP were retained and used to construct the $\mathbf{G}$ matrix and $\mathbf{H}$ matrix.

\section{Estimation of Genetic Parameters}

Genetic parameters were estimated using AIREMLF90 (Misztal et al., 2014) using a pedigree relationship matrix (A), genomic relationship matrix $(\mathbf{G})$, or combined pedigree and genomic relationship matrix $(\mathbf{H})$. Heritabilities were estimated with univariate models. For the pedigree analyses, the univariate model was as follows:

$$
\mathbf{y}=\mathbf{X b}+\mathbf{Z a}+\mathbf{e}
$$

Table 1. Number of records (n), mean, and SD of biomarkers measured in serum and their mid-infrared (MIR)-predicted counterparts ${ }^{1}$

\begin{tabular}{|c|c|c|c|c|c|c|}
\hline \multirow[b]{2}{*}{ Trait } & \multicolumn{2}{|c|}{ A matrix } & \multicolumn{2}{|c|}{$\mathbf{G}$ matrix } & \multicolumn{2}{|c|}{$\mathbf{H}$ matrix } \\
\hline & $\mathrm{n}$ & Mean $\pm \mathrm{SD}$ & $\mathrm{n}$ & Mean $\pm \mathrm{SD}$ & $\mathrm{n}$ & Mean $\pm \mathrm{SD}$ \\
\hline $\mathrm{BHB}^{2}$ & 1,859 & $0.49 \pm 0.24$ & 1,810 & $0.48 \pm 0.22$ & 2,198 & $0.49 \pm 0.23$ \\
\hline $\mathrm{MBHB}^{3}$ & 9,158 & $0.65 \pm 0.23$ & 6,629 & $0.65 \pm 0.22$ & 9,657 & $0.65 \pm 0.23$ \\
\hline Fatty acids & 1,859 & $0.53 \pm 0.32$ & 1,810 & $0.51 \pm 0.32$ & 2,198 & $0.52 \pm 0.31$ \\
\hline $\mathrm{MFA}^{4}$ & 9,158 & $0.55 \pm 0.25$ & 6,629 & $0.57 \pm 0.26$ & 9,657 & $0.55 \pm 0.26$ \\
\hline Urea & 1,859 & $5.62 \pm 1.99$ & 1,810 & $5.57 \pm 1.92$ & 2,198 & $5.53 \pm 1.90$ \\
\hline Murea $^{5}$ & 9,158 & $5.00 \pm 1.80$ & 6,629 & $4.89 \pm 1.77$ & 9,657 & $4.96 \pm 1.79$ \\
\hline
\end{tabular}

${ }^{1}$ For all traits, the unit of measurement is mmol/L. Analyses were done using a pedigree $(\mathbf{A})$, genomic $(\mathbf{G})$, or combined pedigree and genomic $(\mathbf{H})$ relationship matrix

${ }^{2} \mathrm{BHB}$ measured in serum.

${ }^{3}$ MIR-predicted BHB.

${ }^{4} \mathrm{MIR}$-predicted fatty acids.

${ }^{5} \mathrm{MIR}$-predicted urea. 
with vector of phenotypes $\mathbf{y}$ [BHB, fatty acids, urea, MIR-predicted BHB (MBHB), MIR-predicted fatty acids (MFA), and MIR-predicted urea (Murea)], design matrices $\mathbf{X}$ and $\mathbf{Z}$ allocating phenotypes to vectors $\mathbf{b}$ and a containing fixed effects (herd, days in milk, parity, test year, and test month) and additive genetic breeding values, respectively, and a vector of random residuals e. Vectors a and e were distributed as $N\left(0, \mathbf{A} \sigma_{a}^{2}\right)$ and $N\left(0, \mathbf{E} \sigma_{e}^{2}\right)$, with pedigree-based relationship matrix (based on 3 generations of pedigree) $\mathbf{A}$, additive genetic variance $\sigma_{a}^{2}$, diagonal matrix $\mathbf{E}$, and residual error variance $\sigma_{e}^{2}$.

In the genomic analyses, $\mathbf{A}$ was replaced by genomic relationship matrix G; G was constructed following VanRaden (2008):

$$
\mathbf{G}=\frac{\mathbf{Z Z}^{\prime}}{k}
$$

with $k=2 \Sigma p_{j}\left(1-p_{j}\right)$ and $p_{j}$ equal to the allele frequency for variant $j$. In the single step analyses, $\mathbf{A}$ was replaced by relationship matrix $\mathbf{H}$ that combines $\mathbf{A}$ and G (Aguilar et al., 2010) as follows:

$$
\mathbf{H}=\mathbf{A}+\left(\begin{array}{cc}
0 & 0 \\
0 & \mathbf{G}-\mathbf{A}_{22}
\end{array}\right)
$$

where $\mathbf{A}_{22}$ is the part of the pedigree-based relationship matrix containing the genotyped individuals.

Genetic and phenotypic correlations between traits were estimated using the following bivariate model:

$$
\left(\begin{array}{l}
\mathbf{y}_{1} \\
\mathbf{y}_{2}
\end{array}\right)=\left(\begin{array}{cc}
\mathbf{X}_{1} & 0 \\
0 & \mathbf{X}_{2}
\end{array}\right)\left(\begin{array}{l}
\mathbf{b}_{1} \\
\mathbf{b}_{2}
\end{array}\right)+\left(\begin{array}{cc}
\mathbf{Z}_{1} & 0 \\
0 & \mathbf{Z}_{2}
\end{array}\right)\left(\begin{array}{l}
\mathbf{a}_{1} \\
\mathbf{a}_{2}
\end{array}\right)+\left(\begin{array}{l}
\mathbf{e}_{1} \\
\mathbf{e}_{2}
\end{array}\right)
$$

with vectors and matrices $\mathbf{y}_{i}$ (BHB and $\mathrm{MBHB}$, fatty acids and MFA, and urea and Murea), $\mathbf{X}_{i}, \mathbf{f}_{i}, \mathbf{Z}_{i}, \mathbf{a}_{i}$, and $\mathbf{e}_{i}$ similar to those in the univariate model for trait $i$, where $i=1$ was the observed trait and $i=2$ was the MIR-predicted trait. The variance-covariance model used for the bivariate model for the pedigree analyses was as follows:

$$
\operatorname{Var}\left(\begin{array}{l}
\mathbf{a}_{1} \\
\mathbf{a}_{2} \\
\mathbf{e}_{1} \\
\mathbf{e}_{2}
\end{array}\right)=\left(\begin{array}{cccc}
\mathbf{A} \sigma_{a, 1}^{2} & \mathbf{A} \sigma_{a 1, a 2}^{2} & 0 & 0 \\
\mathbf{A} \sigma_{a 1, a 2}^{2} & \mathbf{A} \sigma_{a, 2}^{2} & 0 & 0 \\
0 & 0 & \mathbf{E} \sigma_{e, 1}^{2} & \mathbf{E} \sigma_{e 1, e 2}^{2} \\
0 & 0 & \mathbf{E} \sigma_{e 1, e 2}^{2} & \mathbf{E} \sigma_{e, 2}^{2}
\end{array}\right),
$$

where for trait $i, \sigma_{a, i}^{2}$ was the additive genetic variance, $\sigma_{e, i}^{2}$ was the residual error variance, $\sigma_{a 1, a 2}^{2}$ was the additive covariance between trait 1 and 2 , and $\sigma_{e 1, e 2}^{2}$ was the residual covariance between trait 1 and 2. For the genomic and single step analyses, $\mathbf{A}$ was replaced by $\mathbf{G}$ and $\mathbf{H}$, respectively.

\section{Genomic Prediction}

To estimate the prediction accuracy, the cows with measured biomarkers were randomly divided into 5 subsets for a 5-fold cross-validation. For each of the folds, 4 subsets were used as reference population to predict the breeding values in the remaining subset. Four prediction scenarios were compared: using only measured biomarkers (ST_M), using only predicted biomarkers (ST_P), using measured and predicted biomarkers, and combining measured and predicted biomarkers fitted either as a single trait $\left(\mathbf{S} \mathbf{T}_{-} \mathbf{M}+\mathbf{P}\right)$ or as correlated traits $\left(\mathbf{M T} \_\mathbf{M}+\mathbf{P}\right)$. Measured and prediction biomarkers were standardized to ensure they had equal means and standard deviations for the genomic prediction analyses. The validation populations were the same for all scenarios and contained only cows with measured biomarkers. If a cow had both measured and MIR-predicted biomarkers, we only used the measured biomarker record. Prediction accuracy $r$ was subsequently calculated as the correlation between the predicted breeding values and adjusted phenotypes divided by the square root of the heritability. Genomic prediction was performed using the A-matrix (BLUP), the G-matrix (GBLUP), or the H-matrix (ssGBLUP).

Additionally, we estimated the accuracy for larger reference populations using the estimated prediction accuracies and theoretical formulas (Goddard et al., 2011 ) to determine the number of animals required to have a reasonable level of accuracy for planning future data collection. Following Goddard et al. (2011), the accuracy of genomic prediction was calculated as follows:

$$
r=\sqrt{\frac{\theta}{1+\theta-r^{2} h^{2}}}
$$

where $\theta=\frac{T h^{2}}{M_{e}}, T=$ the size of the reference population, $h^{2}=$ the heritability, and $M_{e}=$ the effective number of independent chromosome segments. While estimating $M_{e}$ can be challenging (Brard and Ricard, 2015; van den Berg et al., 2019), accuracies obtained in cross- 
validation $(r)$ can be used to estimate $\theta_{1}$ for a certain data set as follows:

$$
\theta_{1}=\frac{r^{2}-r^{4} h^{2}}{1-r^{2}}
$$

Subsequently, $\theta_{1}$ was used to estimate the accuracy of a larger reference population, following van den Berg et al. (2019). For a larger reference population, $\theta_{2}$ was estimated as $\theta_{2}=\frac{T_{2}}{T_{1}} \times \theta_{1}$, where $T_{1}$ was the size of the reference population used to estimate $\theta_{1}$, and $T_{2}$ was the size of the larger reference population the size of the reference. The predicted accuracy for reference population $2\left(r^{2}\right)$ was then calculated as follows:

$$
r^{2}=\sqrt{\frac{\theta_{2}}{1+\theta_{2}-r_{2}^{2} h^{2}}} .
$$

This was done for reference populations with a size of 15,$000 ; 20,000 ; 50,000 ; 100,000$; and 200,000. To account for potential error in the estimation of the heritability, we estimated $\theta_{2}$ and $r_{2}$ using the estimated heritability, the estimated heritability plus twice the standard error, and the estimated heritability minus twice the standard error.

\section{RESULTS}

\section{Heritabilities}

Heritabilities for BHB, fatty acids, urea, and their MIR predictions, are shown in Table 2. 0.04 using the A matrix to 0.7 using the $\mathbf{G}$ matrix. Estimates for urea varied from 0.10 using the $\mathbf{A}$ matrix to 0.12 using the G matrix. With standard errors ranging from 0.03 to 0.05, differences in heritabilities obtained with different relationship matrices were not significant for BHB and urea. For fatty acids, however, a large difference was observed between a heritability of $0.21( \pm 0.06)$ estimated using the $\mathbf{A}$ matrix and a heritability of 0.13 ( \pm 0.04 ) using the $\mathbf{G}$ matrix; the $\mathbf{H}$ matrix yielded a heritability of $0.14( \pm 0.04)$, similar to that obtained using the $\mathbf{G}$ matrix. Heritability estimates for MBHB were larger than those of $\mathrm{BHB}$, ranging from 0.10 with the A matrix to 0.12 with the $\mathbf{H}$ matrix, while MFA and Murea had lower heritability estimates than fatty acids and urea, with heritabilities between 0.11 and 0.15 for MFA. Heritability estimates of Murea were similar to those obtained with urea, ranging from 0.08 with the $\mathbf{G}$ matrix to 0.13 with the $\mathbf{A}$ matrix.
Table 2. Heritabilities $( \pm \mathrm{SE})$ of serum biomarkers and their midinfrared (MIR) predictions ${ }^{1}$

\begin{tabular}{lccc}
\hline Trait & A matrix & G matrix & H matrix \\
\hline BHB $^{2}$ & $0.04 \pm 0.04$ & $0.07 \pm 0.04$ & $0.05 \pm 0.03$ \\
MBHB $^{3}$ & $0.10 \pm 0.02$ & $0.11 \pm 0.02$ & $0.12 \pm 0.02$ \\
Fatty acids & $0.21 \pm 0.06$ & $0.13 \pm 0.04$ & $0.14 \pm 0.04$ \\
MFA $^{4}$ & $0.15 \pm 0.02$ & $0.11 \pm 0.02$ & $0.11 \pm 0.01$ \\
Urea $^{\text {Murea }}$ & $0.10 \pm 0.05$ & $0.11 \pm 0.04$ & $0.12 \pm 0.04$ \\
& $0.13 \pm 0.02$ & $0.08 \pm 0.01$ & $0.09 \pm 0.01$ \\
\hline
\end{tabular}

${ }^{1}$ Analyses were done using a pedigree $(\mathbf{A})$, genomic $(\mathbf{G})$, or combined pedigree and genomic $(\mathbf{H})$ relationship matrix.

${ }^{2} \mathrm{BHB}$ measured in serum.

${ }^{3}$ MIR-predicted BHB.

${ }^{4}$ MIR-predicted fatty acids.

${ }^{5}$ MIR-predicted urea.

\section{Genetic Correlations Between Metabolic Traits and Their MIR Predictions}

Genetic correlations between biomarkers and MIR predictions were moderate for BHB and fatty acids, and almost 1 for urea (Table 3). For all traits, genetic correlations were largest using the $\mathbf{A}$ matrix, with correlations of $0.44,0.56$, and 0.98 for $\mathrm{BHB}$, fatty acids, and urea, respectively. Correlations were lower using the $\mathbf{G}$ matrix, while correlations using the $\mathbf{H}$ matrix were in between those obtained with the $\mathbf{A}$ matrix and the $\mathbf{G}$ matrix. For urea, the difference between methods was small and insignificant, and differences for BHB and fatty acids were larger, though with large standard errors. The largest differences were observed for fatty acids, with genetic correlations of $0.56,0.28$, and 0.32 observed for the $\mathbf{A}$ matrix, $\mathbf{G}$ matrix, and $\mathbf{H}$ matrix, respectively. While standard errors were large (0.13-0.15), the difference between the $\mathbf{A}$ matrix and the $\mathbf{G}$ matrix was substantial. Phenotypic correlations were slightly higher than the genetic correlations for $\mathrm{BHB}$, but slightly lower for fatty acids and urea, and similar between all methods (Appendix Table A1).

\section{Genomic Prediction}

Figure 1 compares the genomic prediction accuracies of biomarkers in different scenarios, where we

Table 3. Genetic correlations $( \pm \mathrm{SE})$ between serum biomarkers and their MIR predictions ${ }^{1}$

\begin{tabular}{lccc}
\hline Trait & A matrix & G matrix & H matrix \\
\hline BHB $^{2}$ & $0.44 \pm 0.32$ & $0.25 \pm 0.18$ & $0.35 \pm 0.20$ \\
Fatty acids & $0.56 \pm 0.14$ & $0.28 \pm 0.15$ & $0.32 \pm 0.13$ \\
Urea & $0.98 \pm 0.03$ & $0.96 \pm 0.07$ & $0.97 \pm 0.04$ \\
\hline
\end{tabular}

${ }^{1}$ Analyses were done using a pedigree $(\mathbf{A})$, genomic $(\mathbf{G})$, or combined pedigree and genomic $(\mathbf{H})$ relationship matrix

${ }^{2} \mathrm{BHB}$ measured in serum. 

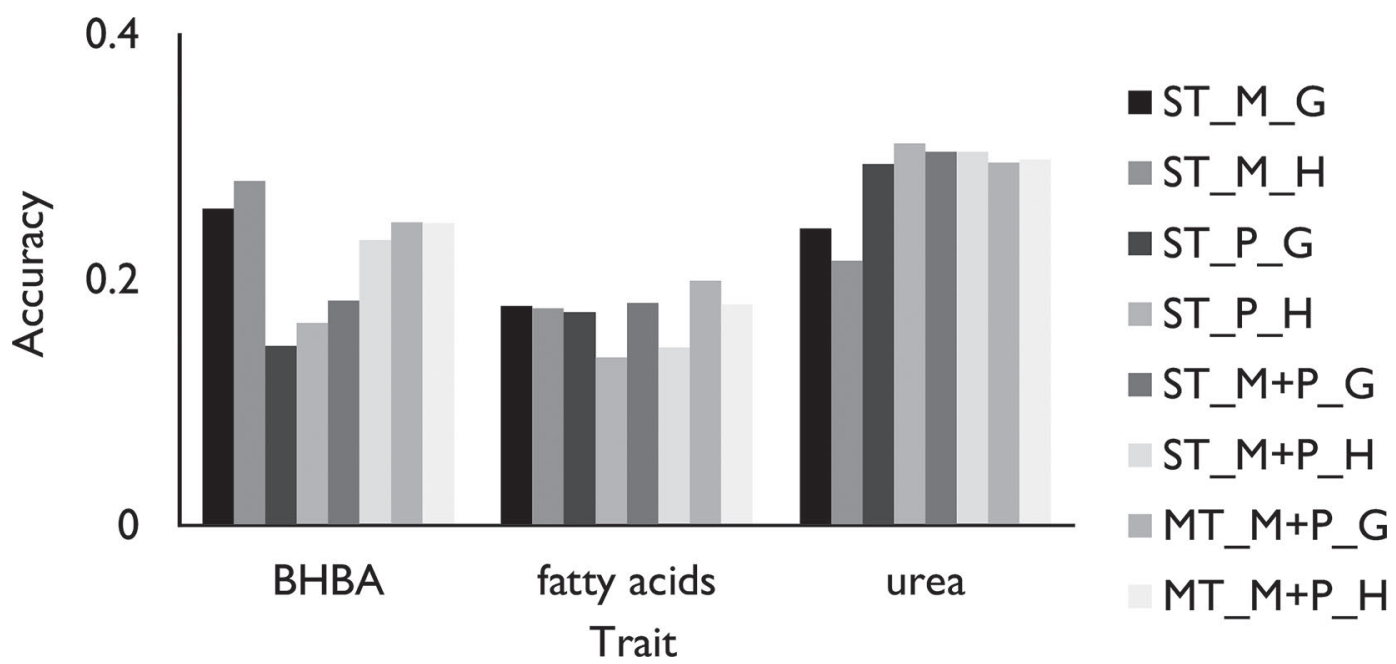

Figure 1. Prediction accuracy estimated in cross-validation. ST_M_G = single trait genomic BLUP (GBLUP) using measured biomarkers; ST_M_H = single trait single step GBLUP (ssGBLUP) using measured biomarkers; ST_M+P_G = single trait GBLUP fitting measured biomarkers and mid-infrared (MIR) predictions as a single trait; ST_M+P_H = single trait ssGBLUP fitting measured biomarkers and MIR predictions as a single trait; $\mathrm{MT} \_\mathrm{M}+\mathrm{P} \_\mathrm{G}=$ multitrait GBLUP fitting measured biomarkers and $\mathrm{MIR}$ predictions as correlated traits; MT_M+P_H $=$ multitrait ssGBLUP fitting measured biomarkers and MIR predictions as correlated traits. BHB $=\beta$-hydroxybutyric acid.

varied the prediction models and the inclusion of MIRpredicted traits. Including MIR-predicted traits in the reference population increased prediction accuracies for urea, but decreased prediction accuracy for BHB. For fatty acids, differences in accuracies between scenarios were minimal. Appendix Table A2 shows the correlations between predicted breeding values and adjusted phenotypes per cross-validation fold.

For BHB, ssGBLUP yielded slightly higher accuracies than GBLUP, and the multitrait models yielded higher accuracies than the single trait models that included MIR-predicted BHB. Using only measured BHB, the accuracy was 0.26 and 0.28 for single trait GBLUP (ST_M_G) and single trait ssGBLUP (ST_M_H), respectively. Using only predicted BHB resulted in accuracies of 0.15 and 0.17 for ST_P_G and ST_P_H. Merging BHB and MBHB in a single trait $\left(\mathbf{S} \mathbf{T}_{-} \mathbf{M}+\mathbf{P}\right)$ reduced prediction accuracies compared with only using measured BHB. Including MIR-predicted BHB as a correlated trait in the model (MT_M+P) decreased the accuracy to 0.25 for both multitrait GBLUP (MT_M+P_G) and multitrait ssGBLUP (MT_M+P_H).

For fatty acids, differences between scenarios were smaller than for the other traits, varying from 0.14 for ST_P_H to 0.20 for MT_M+P_G. Using the $\mathbf{H}$ matrix resulted in lower accuracies for fatty acids, especially for the scenarios including MIR-predicted fatty acids. For urea, accuracies of 0.24 and 0.22 were obtained with ST_M_G and ST_M_H, respectively. Contrarily to BHB and fatty acids, using only MIR-predicted urea resulted in a higher accuracy (0.29 for ST_P_G and 0.31 for ST_P_H) than using only using measured urea. All scenarios that combined measured and MIRpredicted urea (both single trait and multitrait, using either $\mathbf{G}$ matrix or $\mathbf{H}$ matrix) resulted in an accuracy of 0.30 .

Figure 2 shows an estimate of the increase in prediction accuracy to be expected for urea, using ssGBLUP for the estimated heritability (0.12) and upper and lower limit of the $95 \%$ confidence interval of the estimate $(0.20$ and 0.14$)$. To obtain an accuracy of 0.6 , a reference population between 30,000 and 150,000 cows would be required, and between 90,000 and >200,000 cows for an accuracy of 0.8 . Applying the formulas to the heritability and accuracy obtained for GBLUP resulted in similar numbers (results not shown).

\section{DISCUSSION}

In our study, we showed that Murea can increase the accuracy of genomic prediction of serum urea. Murea is heritable, has a genetic correlation close to 1 with urea, and is available on a larger number of individuals than urea. In a small cross-validation study, using Murea instead of or in addition to measured urea resulted in increased prediction accuracies for urea. Estimates of genetic correlations between $\mathrm{MBHB}$ and $\mathrm{BHB}$, and MFA and fatty acids were lower and had large standard errors; therefore, further research with a larger number of records may be required to assess the potential use of MBHB and MFA for genomic prediction. 


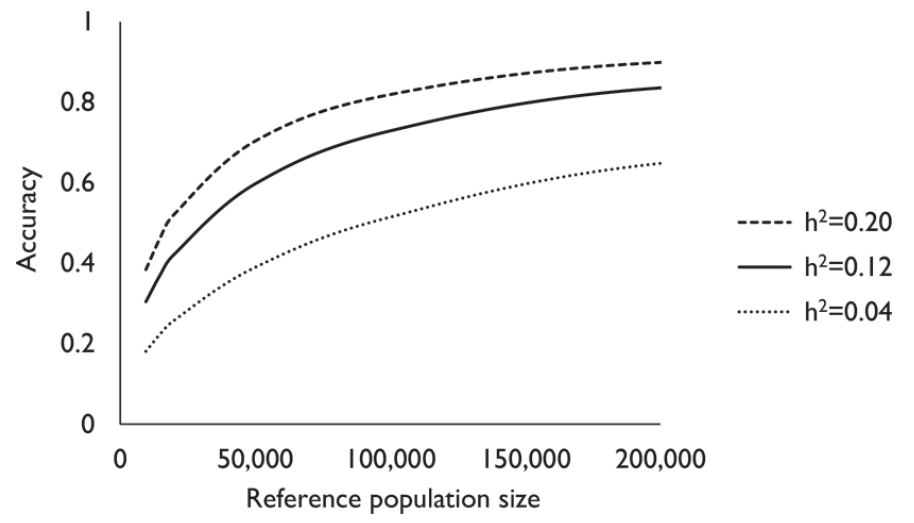

Figure 2. Prediction accuracy as a function of the size of the reference population and heritability $\left(\mathrm{h}^{2}\right)$. Heritabilities of $0.12,0.04$, and 0.20 correspond to the estimated $h^{2}$ of urea, the estimated $h^{2}$ minus twice the standard error, and the estimated $\mathrm{h}^{2}$ plus twice the standard error, respectively.

\section{Heritabilities}

For all traits, heritabilities were low to moderate, and significantly greater than zero. Estimates obtained with the $\mathbf{G}$ matrix were lower than those published by Luke et al. (2019a), although the differences were not significant. The cows in the analyses of Luke et al. (2019a) were included in our data; hence, the differences observed illustrate the uncertainty of estimates of genetic parameters using small datasets. Other studies have reported various estimates for the heritability of BHB, ranging from 0.07 (Tsiamadis et al., 2016) to 0.40 (Oikonomou et al., 2008). Benedet et al. (2020) reported a heritability of $\mathrm{MBHB}$ of 0.21 , higher than the heritability of 0.10 to 0.12 obtained in our study, and a slightly larger heritability for MFA [0.14 in the study by Benedet et al. (2020) and 0.11 in our study].

Heritabilities of MBHB were higher than heritabilities of measured BHB, whereas heritabilities of MFA were smaller than heritabilities for fatty acids, and heritabilities estimates for urea and Murea were sometimes larger but sometimes lower, depending on the relationship matrix. McParland et al. (2015) observed lower heritabilities for MIR-predicted energy status than measured energy status, whereas Cecchinato et al. (2009) reported higher heritabilities for MIR-predicted coagulation property than for measured milk coagulation property. For most trait-method combinations, differences in heritability were small and can be explained by the differences in data sets. Estimates for measured traits had relatively large standard errors (between 0.03 and 0.06) due to the small data size. For almost all traits and methods, the $95 \%$ confidence intervals of the heritability estimate would overlap between measured and predicted traits. For example, the largest difference observed was between fatty acids and MFA, with heritabilities of 0.21 and 0.15 , respectively. With standard errors of 0.06 and $0.02,95 \%$ confidence intervals would be 0.09 to 0.33 and 0.11 to 0.19 for fatty acids and MFA, respectively.

Differences in heritabilities obtained with different relationship matrices were inconsistent and not significant. The largest difference was observed for fatty acids, where the $\mathbf{A}$ matrix yielded a heritability of 0.21 , and the $\mathbf{G}$ matrix a heritability of 0.13 . However, with standard errors of 0.06 and 0.04 , the difference was not significant. Several studies have compared heritabilities using different relationship matrices for various traits and species. In some studies, heritabilities were higher using a genomic relationship matrix or combined genomic and pedigree matrix (Raidan et al., 2018; Wang et al., 2020), while Veerkamp et al. (2011) reported higher heritabilities using only pedigree information, and other studies showed different results for different traits (Momen et al., 2017; Hidalgo et al., 2020).

\section{Genetic Correlations}

Correlations between urea and Murea were very strong and almost 1; this suggests that, from a genetic perspective, urea and Murea can be considered as the same trait. Genetic correlations between BHB and MBHB (0.25-0.44) and between fatty acids and MFA (0.28-0.56) were moderate and had large standard errors $(0.13-0.32)$. The genetic correlations are in line with the prediction accuracies of the MIR equations reported by Ho et al. (2020): prediction accuracies of the MIR equations were highest for urea and lowest for fatty acids. Despite having a genomic correlation significantly lower than 1, MBHB and MFA may still be useful for genomic prediction, especially if many more records would be available for MBHB and MFA than for BHB and fatty acids. This may be possible through commercial herd-testing. However, herd-testing is usually not done in early lactation, limiting the number of MIR records available for analysis of biomarkers during early lactation. Further studies with a larger data set, especially with a larger number of records for BHB and fatty acids, will be required to more accurately estimate correlations between $\mathrm{MBHB}$ and $\mathrm{BHB}$, and MFA and fatty acids and assess the potential use of MBHB and MFA for genomic prediction. Additionally, further improvement of the MIR prediction equations may result in a higher prediction accuracy of the MIR equations, and consequently, higher genetic correlations between BHB and MBHB, and fatty acids and MFA.

Genetic correlations were lower using the $\mathbf{G}$ matrix than using the $\mathbf{A}$ matrix, with differences between 0.02 and 0.28 . Although none of these differences were 
significant because of the large standard errors, the consistent trend of lower heritabilities and lower genetic correlations suggest that the $\mathbf{G}$ matrix does captures less of the genetic covariance than pedigree information. Similar to the heritability estimates, several studies report differences in genetic correlations based on the information used to construct the relationship matrix used (Veerkamp et al., 2011; Momen et al., 2017; Hidalgo et al., 2020), but there is no clear consensus on the optimal method.

\section{Genomic Prediction}

For ST_M_G, prediction accuracies were lower than the accuracies published by Luke et al. (2019a). The accuracy of genomic prediction increases when the size of the reference population increases (Goddard, 2009); therefore, we expected higher prediction accuracies in our study than in the study by Luke et al. (2019a), due to the larger reference population. However, prediction accuracies in our study were lower. This may be due to the small size of the data set, and accuracies can be influenced by slight differences in, for example, relatedness between the reference and validation populations.

Including MIR predictions improved prediction accuracies for urea, but not for BHB and only minimal for fatty acids. This was not surprising, given the large genetic correlation between urea and Murea, and the smaller genetic correlations between $\mathrm{BHB}$ and $\mathrm{MBHB}$ and fatty acids and MFA. For urea, prediction accuracies were similar in all scenarios that included Murea, even in the scenarios that did not include any measured urea in the reference population. This further confirms the potential for Murea to be used to select for urea.

In dairy cattle, reference and validation populations are commonly constructed based on the date of birth of cows. This mimics the situation where older bulls and cows have records, while breeding values are predicted for young selection candidates without phenotypes. However, because of the small number of cows with records for biomarkers, we performed a 5-fold crossvalidation instead. Because of this, relatedness between reference and validation populations in our study may have been different than in practice, and the large differences in correlation between folds indicated the sensitivity of the accuracy to the composition of the reference and validation populations. We did not find a clear explanation for the differences observed: all crossvalidation folds had very similar phenotypic means and standard deviations for all traits, and a principal component analysis of the genotypes did not show any clustering. A larger data set will be necessary to confirm our findings and potentially identify explanations for the observed differences.
While the increase in genomic prediction accuracy due to the inclusion of MIR-predicted biomarkers is promising, higher prediction accuracies than obtained in this study would be desirable for implementation of genomic prediction. Therefore, we applied theoretical formulas to have an idea of the size of the reference population required for higher accuracies. As shown in Figure 2, a reference population of at least 30,000 to 150,000 cows would be required for an accuracy of 0.6. Reference populations of 30,000 or more cows with measured biomarkers are not feasible in the near future; therefore, using MIR-predicted biomarkers may provide a promising alternative, and this approach appears to be especially well suited to serum urea.

\section{CONCLUSIONS}

Our results show that biomarkers measured in serum and their MIR predictions are heritable and are significantly genetically correlated, albeit with high standard errors for BHB and urea. For all traits, including MIR data resulted in increases in genomic prediction accuracy for urea, but not for BHB or fatty acids. Genetic correlations were particularly high between urea and Murea, demonstrating the potential of using Murea for genomic prediction of urea. For BHB and fatty acids, further studies with larger data sets are required to obtain more accurate estimates of genetic correlations.

\section{ACKNOWLEDGMENTS}

This study was undertaken as part of the DairyBio program, which is jointly funded by Dairy Australia (Melbourne, Australia) and Agriculture Victoria (Melbourne, Australia), and The Gardiner Foundation (Melbourne, Australia). The authors thank Di Mapleson, Brigid Ribaux, and the staff at Ellinbank Dairy Research Centre (Ellinbank, Australia) for their technical expertise and assistance, Erika Oakes and Michelle Axford of Datagene (Bundoora, Australia) for their work coordinating this study, and the farmers who participated in this project. Hico Pty Ltd. (Maffra, Victoria, Australia) and TasHerd Pty Ltd. (Hadspen, Australia) are gratefully acknowledged for collecting milk samples and providing MIR spectral data. Regional Laboratory Services (Benalla, Victoria, Australia) are thanked for measuring the concentrations of serum biomarkers used in this study. The authors extend their gratitude to the AgriBio molecular genetics team (Bundoora, Australia), particularly Coralie Reich, Brett Mason, and Amanda Chamberlain, for genotyping. Special thanks to Iona MacLeod (Bundoora, Australia) for her advice on imputation of genotypes, and the farmers and Datagene (Bundoora, Australia) for access to data used in 
this study. The authors have not stated any conflicts of interest.

\section{REFERENCES}

Aguilar, I., I. Misztal, D. L. Johnson, A. Legarra, S. Tsuruta, and T. J. Lawlor. 2010. Hot topic: A unified approach to utilize phenotypic, full pedigree, and genomic information for genetic evaluation of Holstein final score. J. Dairy Sci. 93:743-752. https://doi.org/10 $.3168 /$ jds.2009-2730.

Belay, T. K., B. S. Dagnachew, Z. M. Kowalski, and T. Ådnøy. 2017. An attempt at predicting blood $\beta$-hydroxybutyrate from Fouriertransform mid-infrared spectra of milk using multivariate mixed models in Polish dairy cattle. J. Dairy Sci. 100:6312-6326. https:/ /doi.org/10.3168/jds.2016-12252.

Benedet, A., A. Costa, M. De Marchi, and M. Penasa. 2020. Heritability estimates of predicted blood $\beta$-hydroxybutyrate and nonesterified fatty acids and relationships with milk traits in early-lactation Holstein cows. J. Dairy Sci. 103:6354-6363. https://doi.org/10 .3168/jds.2019-17916

Boichard, D., and M. Brochard. 2012. New phenotypes for new breeding goals in dairy cattle. Animal 6:544-550. https://doi.org/10 $.1017 / \mathrm{S} 1751731112000018$.

Brard, S., and A. Ricard. 2015. Is the use of formulae a reliable way to predict the accuracy of genomic selection? J. Anim. Breed. Genet. 132:207-217. https://doi.org/10.1111/jbg.12123.

Butler, W. R., J. J. Calaman, and S. W. Beam. 1996. Plasma and milk urea nitrogen in relation to pregnancy rate in lactating dairy cattle. J. Anim. Sci. 74:858. https://doi.org/10.2527/1996.744858x.

Cecchinato, A., M. de Marchi, L. Gallo, G. Bittante, and P. Carnier. 2009. Mid-infrared spectroscopy predictions as indicator traits in breeding programs for enhanced coagulation properties of milk. J. Dairy Sci. 92:5304-5313. https://doi.org/10.3168/jds.2009-2246.

De Marchi, M., V. Toffanin, M. Cassandro, and M. Penasa. 2014. Invited review: Mid-infrared spectroscopy as phenotyping tool for milk traits1. J. Dairy Sci. 97:1171-1186. https://doi.org/10.3168/ jds.2013-6799.

Goddard, M. 2009. Genomic selection: Prediction of accuracy and maximisation of long term response. Genetica 136:245-257. https: //doi.org/10.1007/s10709-008-9308-0.

Goddard, M. E., B. J. Hayes, and T. H. E. Meuwissen. 2011. Using the genomic relationship matrix to predict the accuracy of genomic selection. J. Anim. Breed. Genet. 128:409-421. https://doi.org/10 .1111/j.1439-0388.2011.00964.x.

Grelet, C., A. Vanlierde, M. Hostens, L. Foldager, M. Salavati, K. L. Ingvartsen, M. Crowe, M. T. Sorensen, E. Froidmont, C. P. Ferris, C. Marchitelli, F. Becker, T. Larsen, F. Carter, and F. Dehareng. 2019. Potential of milk mid-IR spectra to predict metabolic status of cows through blood components and an innovative clustering approach. Animal 13:649-658. https://doi.org/10.1017/ S1751731118001751.

Hidalgo, J., S. Tsuruta, D. Lourenco, Y. Masuda, Y. Huang, K. A. Gray, and I. Misztal. 2020. Changes in genetic parameters for fitness and growth traits in pigs under genomic selection. J. Anim. Sci. 98:skaa032. https://doi.org/10.1093/jas/skaa032.

Ho, P. N., T. D. W. Luke, and J. E. Pryce. 2020. Validation of milk mid-infrared spectroscopy for predicting the metabolic status of lactating dairy cows in Australia. J. Dairy Sci. 104. https://doi .org/10.3168/jds.2020-19603.

Luke, T. D. W., T. T. T. Nguyen, S. Rochfort, W. J. Wales, C. M. Richardson, M. Abdelsayed, and J. E. Pryce. 2019a. Genomic prediction of serum biomarkers of health in early lactation. J. Dairy Sci. 102:11142-11152. https://doi.org/10.3168/jds.2019-17127.

Luke, T. D. W., S. Rochfort, W. J. Wales, V. Bonfatti, L. Marett, and J. E. Pryce. 2019b. Metabolic profiling of early-lactation dairy cows using milk mid-infrared spectra. J. Dairy Sci. 102:1747-1760. https://doi.org/10.3168/jds.2018-15103.

McParland, S., E. Kennedy, E. Lewis, S. G. Moore, B. McCarthy, M. O'Donovan, and D. P. Berry. 2015. Genetic parameters of dairy cow energy intake and body energy status predicted using mid- infrared spectrometry of milk. J. Dairy Sci. 98:1310-1320. https:/ /doi.org/10.3168/jds.2014-8892.

Mevik, B. H., and R. Wehrens. 2007. The pls package: Principal component and partial least squares regression in R. J. Stat. Softw. 18. https://doi.org/10.18637/jss.v018.i02.

Misztal, I., D. Lourenco, and A. Legarra. 2020. Current status of genomic evaluation. J. Anim. Sci. 98:skaa101. https://doi.org/10 $.1093 /$ jas/skaa101.

Misztal, I., S. Tsuruta, D. Lourenco, I. Aguilar, A. Legarra, and Z. Vitezica. 2014. Manual for BLUPF90 family of programs. http:// nce.ads.uga.edu/wiki/lib/exe/fetch.php?media=blupf90 all2.pdf.

Momen, M., A. A. Mehrgardi, A. Sheikhy, A. Esmailizadeh, M. A. Fozi, A. Kranis, B. D. Valente, G. J. M. Rosa, and D. Gianola. 2017. A predictive assessment of genetic correlations between traits in chickens using markers. Genet. Sel. Evol. 49:16. https:// doi.org/10.1186/s12711-017-0290-9.

NHMRC (National Health and Medical Research Council). 2013. Australian Code for the Care and Use of Animals for Scientific Purposes. 8th ed. NHMRC Publications, Canberra, Australia.

Oikonomou, G., G. E. Valergakis, G. Arsenos, N. Roubies, and G. Banos. 2008. Genetic profile of body energy and blood metabolic traits across lactation in primiparous Holstein cows. J. Dairy Sci. 91:2814-2822. https://doi.org/10.3168/jds.2007-0965.

Ospina, P. A., D. V. Nydam, T. Stokol, and T. R. Overton. 2010a. Associations of elevated nonesterified fatty acids and $\beta$-hydroxybutyrate concentrations with early lactation reproductive performance and milk production in transition dairy cattle in the northeastern United States. J. Dairy Sci. 93:1596-1603. https: //doi.org/10.3168/jds.2009-2852.

Ospina, P. A., D. V. Nydam, T. Stokol, and T. R. Overton. 2010b. Evaluation of nonesterified fatty acids and $\beta$-hydroxybutyrate in transition dairy cattle in the northeastern United States: Critical thresholds for prediction of clinical diseases. J. Dairy Sci. 93:546554. https://doi.org/10.3168/jds.2009-2277.

Patry, C., and V. Ducrocq. 2011. Evidence of biases in genetic evaluations due to genomic preselection in dairy cattle. J. Dairy Sci. 94:1011-1020. https://doi.org/10.3168/jds.2010-3804.

Pralle, R. S., and H. M. White. 2020. Symposium review: Big data, big predictions: Utilizing milk Fourier-transform infrared and genomics to improve hyperketonemia management. J. Dairy Sci. 103:3867-3873. https://doi.org/10.3168/jds.2019-17379.

Pryce, J. E., K. L. Parker Gaddis, A. Koeck, C. Bastin, M. Abdelsayed, N. Gengler, F. Miglior, B. Heringstad, C. Egger-Danner, K. F. Stock, A. J. Bradley, and J. B. Cole. 2016. Invited review: Opportunities for genetic improvement of metabolic diseases. J. Dairy Sci. 99:6855-6873. https://doi.org/10.3168/jds.2016-10854.

Raboisson, D., A. Albaaj, G. Nonne, and G. Foucras. 2017. High urea and pregnancy or conception in dairy cows: A meta-analysis to define the appropriate urea threshold. J. Dairy Sci. 100:7581-7587. https://doi.org/10.3168/jds.2016-12009.

Raidan, F. S. S., L. R. Porto-Neto, Y. Li, S. A. Lehnert, and A. Reverter. 2018. Weighting genomic and genealogical information for genetic parameter estimation and breeding value prediction in tropical beef cattle. J. Anim. Sci. 96:612-617. https://doi.org/10 $.1093 / \mathrm{jas} / \mathrm{skx} 027$.

Sargolzaei, M., J. P. Chesnais, and F. S. Schenkel. 2014. A new approach for efficient genotype imputation using information from relatives. BMC Genomics 15:478. https://doi.org/10.1186/1471 $-2164-15-478$.

Tsiamadis, V., G. Banos, N. Panousis, M. Kritsepi-Konstantinou, G. Arsenos, and G. E. Valergakis. 2016. Genetic parameters of subclinical macromineral disorders and major clinical diseases in postparturient Holstein cows. J. Dairy Sci. 99:8901-8914. https://doi .org/10.3168/jds.2015-10789.

van den Berg, I., T. H. E. Meuwissen, I. M. MacLeod, and M. E. Goddard. 2019. Predicting the effect of reference population on the accuracy of within, across, and multibreed genomic prediction. J. Dairy Sci. 102:3155-3174. https://doi.org/10.3168/jds.2018-15231.

VanRaden, P. M. 2008. Efficient methods to compute genomic predictions. J. Dairy Sci. 91:4414-4423. https://doi.org/10.3168/jds .2007-0980. 
Veerkamp, R. F., H. A. Mulder, R. Thompson, and M. P. L. Calus. 2011. Genomic and pedigree-based genetic parameters for scarcely recorded traits when some animals are genotyped. J. Dairy Sci. 94:4189-4197. https://doi.org/10.3168/jds.2011-4223.

Wang, L., L. L. Janss, P. Madsen, J. Henshall, C. H. Huang, D. Marois, S. Alemu, A. C. Sørensen, and J. Jensen. 2020. Effect of genomic selection and genotyping strategy on estimation of variance components in animal models using different relationship matrices. Genet. Sel. Evol. 52:31. https://doi.org/10.1186/s12711-020-00550 $-\mathrm{w}$.

\section{ORCIDS}

I. van den Berg (๑ https://orcid.org/0000-0002-9292-8636

P. N. Ho ® https://orcid.org/0000-0001-9481-9612

T. D. W. Luke @ https://orcid.org/0000-0001-5070-2332

J. E. Pryce (ํ) https://orcid.org/0000-0002-1397-1282

\section{APPENDIX}

Table A1. Phenotypic correlations between serum biomarkers and their mid-infrared predictions; analyses were done using a pedigree $(\mathbf{A})$, genomic $(\mathbf{G})$, or combined pedigree and genomic $(\mathbf{H})$ relationship matrix

\begin{tabular}{lccc}
\hline Trait & A matrix & G matrix & H matrix \\
\hline BHB $^{1}$ & $0.59 \pm 0.02$ & $0.57 \pm 0.02$ & $0.58 \pm 0.02$ \\
Fatty acids & $0.43 \pm 0.02$ & $0.45 \pm 0.02$ & $0.43 \pm 0.02$ \\
Urea & $0.87 \pm 0.01$ & $0.86 \pm 0.01$ & $0.87 \pm 0.00$ \\
\hline
\end{tabular}

${ }^{1}$ BHB measured in serum.

Table A2. Correlations between predicted breeding values and phenotypes

\begin{tabular}{|c|c|c|c|c|c|c|c|}
\hline \multirow[b]{2}{*}{ Trait } & \multirow[b]{2}{*}{ Model $^{1}$} & \multicolumn{5}{|c|}{ Cross-validation fold } & \multirow[b]{2}{*}{ Average } \\
\hline & & 1 & 2 & 3 & 4 & 5 & \\
\hline \multirow[t]{8}{*}{ BHB } & ST_M_G ${ }^{2}$ & 0.06 & 0.04 & 0.10 & 0.03 & 0.12 & 0.07 \\
\hline & ST_M_H ${ }^{3}$ & 0.04 & 0.03 & 0.10 & 0.01 & 0.12 & 0.06 \\
\hline & ST_P_G ${ }^{4}$ & 0.04 & 0.05 & 0.04 & 0.02 & 0.05 & 0.04 \\
\hline & ST_P_H ${ }^{5}$ & 0.03 & 0.05 & 0.03 & 0.04 & 0.03 & 0.04 \\
\hline & $\mathrm{ST} \_\mathrm{M}+\mathrm{P} \_\mathrm{G}^{6}$ & 0.03 & 0.07 & 0.07 & 0.01 & 0.07 & 0.05 \\
\hline & $\mathrm{ST} \_\mathrm{M}+\mathrm{P} \_\mathrm{H}^{7}$ & 0.01 & 0.07 & 0.06 & 0.04 & 0.07 & 0.05 \\
\hline & MT_M+P_G ${ }^{8}$ & 0.05 & 0.08 & 0.08 & 0.01 & 0.11 & 0.07 \\
\hline & $\mathrm{MT} \_\mathrm{M}+\mathrm{P} \_\mathrm{H}^{9}$ & 0.04 & 0.08 & 0.06 & 0.00 & 0.09 & 0.05 \\
\hline \multirow[t]{8}{*}{ Fatty acids } & ST_M_G & 0.07 & 0.09 & 0.07 & -0.01 & 0.09 & 0.07 \\
\hline & ST_M_H & 0.06 & 0.07 & 0.07 & 0.07 & 0.02 & 0.10 \\
\hline & ST_P_G & 0.07 & 0.02 & 0.06 & 0.08 & 0.09 & 0.07 \\
\hline & ST_P_H & 0.06 & 0.05 & -0.02 & 0.06 & 0.07 & 0.09 \\
\hline & ST_M+P_G & 0.07 & 0.03 & 0.09 & 0.04 & 0.10 & 0.07 \\
\hline & ST_M+P_H & 0.07 & 0.06 & 0.00 & 0.07 & 0.05 & 0.10 \\
\hline & MT_M+P_G & 0.07 & 0.09 & 0.11 & -0.01 & 0.10 & 0.07 \\
\hline & MT_M+P_H & 0.05 & 0.05 & 0.11 & 0.02 & 0.11 & 0.07 \\
\hline \multirow[t]{8}{*}{ Urea } & ST_M_G & 0.12 & 0.14 & 0.07 & 0.11 & -0.02 & 0.08 \\
\hline & ST_M_H & 0.10 & 0.14 & 0.04 & 0.13 & -0.04 & 0.07 \\
\hline & ST_P_G & 0.09 & 0.20 & 0.10 & 0.09 & 0.02 & 0.10 \\
\hline & ST_P_H & 0.10 & 0.19 & 0.09 & 0.10 & 0.07 & 0.11 \\
\hline & ST_M+P_G & 0.10 & 0.21 & 0.10 & 0.08 & 0.02 & 0.10 \\
\hline & ST_M+P_H & 0.11 & 0.18 & 0.09 & 0.09 & 0.07 & 0.11 \\
\hline & MT_M+P_G & 0.10 & 0.20 & 0.09 & 0.09 & 0.01 & 0.10 \\
\hline & MT_M+P_H & 0.11 & 0.18 & 0.08 & 0.10 & 0.05 & 0.10 \\
\hline
\end{tabular}

${ }^{1}$ ST_M_G = single trait genomic BLUP (GBLUP), using measured biomarkers; ST_M_H = single trait single step GBLUP (ssGBLUP), using measured biomarkers; ST_P_G = single trait GBLUP, using mid-infrared (MIR)-predicted biomarkers; ST_P_G = single trait ssGBLUP, using MIR-predicted biomarkers; ST_M+P_G = single trait GBLUP, fitting measured biomarkers and MIR predictions as a single trait; ST_M+P_H = single trait ssGBLUP, fitting measured biomarkers and MIR predictions as a single trait; $M T \_M+P \_G=$ multitrait GBLUP, fitting measured biomarkers and MIR predictions as correlated traits; and $\mathrm{MT} \_\mathrm{M}+\mathrm{P} \_\mathrm{H}=$ multitrait GBLUP, fitting measured biomarkers and MIR predictions as correlated traits. 